\title{
Les possibilités d'actions de réglage pour assurer l'équilibre de ce marché à l'avenir
}

\author{
Claudine Mathieu Thiébauda, \\ Riccardo Crivelli ${ }^{b}$ \\ a Spécialiste en néphrologie et \\ médecine interne, Lausanne \\ b Economiste, Biasca
}

\section{Un marché comme un autre?}

Le marché des soins et des prestataires de soins, dont font partie les médecins, est-il un marché comme un autre? En Suisse, la santé est un droit pour tous, fixé dans la loi et réglementé par la loi sur l'assurancemaladie qui repose sur le principe de la «libre concurrence régulée» [1]. On reconnaît déjà dans cette formulation la complexité hybride de l'organisation du système de santé suisse.

\section{La demande de la ressource «médecins»}

Dans la réalité du marché de la santé, les acheteurs/ consommateurs, qui devraient déterminer la demande, sont les patients. Ces derniers ont des besoins lorsque leur état de santé est menacé. La demande de prestations de biens et de services de santé naît de ce besoin; ce point est essentiel, car ce besoin est en grande partie imprévisible et non choisi. Le patient va demander de l'information et des soins. La courbe de demande dans le domaine de la santé est peu élastique, c'est-à-dire varie peu, car elle dépend de l'état de santé des patients et non pas de la variation des prix, comme dans un marché concurrentiel.

En ce qui concerne la demande de prestations de santé qui s'adresse aux médecins, on ne peut pas simplement s'en remettre au «libre marché». Le patient manque en général d'informations, que l'offreur, c'està-dire le médecin, détient. C'est donc le plus souvent le médecin qui décide de la prestation de service de santé à laquelle le patient aura finalement recours. Même s'il y a, dans la majorité des cas, une asymétrie évidente concernant l'information, et même si le médecin pouvait être enclin à poursuivre d'autres buts que seulement celui de soigner, il ne faut pas oublier qu'un professionnel de la santé est soumis à des obligations et des bonnes pratiques professionnelles, résumées sous le terme de déontologie ou d'éthique médicale.

La relation médecin-patient a évolué ces dernières années vers une relation dite «informative» voire «délibérative», situation où le patient bien informé devient de plus en plus co-auteur des choix qui s'offrent à lui et acquiert la responsabilité de l'acheteur-demandeur que la théorie de l'induction lui nie [2].

\section{Correspondance:}

Dr Claudine Mathieu Thiébaud 57, avenue Louis-Ruchonnet CH-1003 Lausanne

clm.mathieuth[at]gmail.com

\section{De quels facteurs dépend la demande en médecins?}

La demande en médecins est influencée par les facteurs suivants:
- par les caractéristiques épidémiologiques et démographiques, la pyramide des âges;

- par le niveau socio-économique et éducatif des patients;

- par l'environnement et les conditions de vie;

- par les valeurs «normales» que la société et le monde médical spécialisé définissent: quelle est la frontière entre santé et maladie dans une société ultra-médicalisée?

- par l'évolution constante des techniques médicales, l'extension des campagnes de dépistage et la généralisation des tests diagnostiques [3];

- par le niveau d'information, de la culture sanitaire de la population [1].

Finalement, le pas de la «demande» vers la «consommation» de services de santé peut être influencé par l'offre, selon la théorie de l'induction de la demande par l'offre et partiellement par le prix, en fonction du montant que le patient doit payer de sa poche versus le montant couvert par son assurance maladie sociale ou privée.

\section{Comment prévoir la demande en médecins?}

Sur le plan national, comment prévoir la demande en médecins dans les années à venir, aussi bien en généralistes qu'en spécialistes? Plusieurs paramètres vont influencer cette demande dans l'avenir:

- la croissance et le vieillissement de la population (espérance de vie en Suisse 82,2 ans selon les chiffres de l'OCDE de 2010, moyenne pour les pays de l'OCDE 79,4 ans) vont augmenter la demande; il faut néanmoins souligner que le vieillissement est un facteur qui influence de manière modérée l'augmentation des dépenses de santé: avec l'allongement de l'espérance de vie, le nombre de personnes âgées augmente très logiquement, mais ces dernières sont aussi davantage en bonne santé!

- le mode de vie de nos sociétés occidentales a vu naître une "épidémie» de l'obésité, qui ne cesse de s'aggraver ces dernières années (en Suisse, progression de l'obésité de 5,4\% en 1992 à 8,1\% en 2008, taux moyen de l'obésité dans les pays de l'OCDE $14,9 \%$, chiffres OCDE) avec une cascade de conséquences médicales néfastes, dont le diabète (prévision pour 2030: 552 millions de diabétiques dans le monde, ce qui correspond à 7-8\% de la population mondiale selon les chiffres de la Fédé- 
ration internationale du diabète), les maladies cardio-vasculaires et l'insuffisance rénale.

- l'augmentation du nombre de cancers: l'augmentation de leur incidence est en partie liée à l'évolution de la technologie médicale: on ne détecte pas seulement davantage de tumeurs, on les diagnostique aussi à un stade plus précoce.

Cette analyse suggère qu'il ne faut pas seulement une adaptation du nombre de généralistes, mais avec la complexité des pathologies, il faudra aussi disposer de spécialistes capables de prendre en charge de nouveaux domaines très spécialisés de la médecine.

\section{Pendant dix ans, toute une génération de jeunes médecins n'ont pas eu d'autres choix que de chercher une voie de spécialisation intrahospitalière.}

Nombre de médecins actifs en Suisse et comparaison avec l'OCDE: Selon les chiffres de l'OCDE de 2008, la Suisse compte 3,8 médecins par millier d'habitants, un chiffre plus élevé que la moyenne de 3,2 dans les pays de l'OCDE. Le nombre de généralistes, par contre, est de seulement 0,6 pour 1000 habitants comparé à 1,6 par millier d'habitants en France. La Suisse a donc trop peu de généralistes et un excédent de spécialistes en comparaison avec les pays de l'OCDE.

Statistique médicale de la FMH: Selon les statistiques médicales de 2011 de la FMH [4], le nombre total des médecins exerçant en Suisse a connu une augmentation de 1,9\% comparée à l'année précédente. Cette progression est due surtout à l'augmentation du nombre de femmes médecins $(+4,3 \%$ vs $+0,6 \%$ chez les hommes).

$52,6 \%$ des médecins sont actifs dans le secteur

4 Kraft E. 30849 Médecins au service de la Suisse. Bull Méd Suisses. 2012;93(11):399-403.

5 www.seco.admin.ch ambulatoire. $60,1 \%$ sont des spécialistes, $38,6 \%$ des spécialistes en médecine interne générale (1,3\% sans titre mentionné). La répartition entre spécialistes et

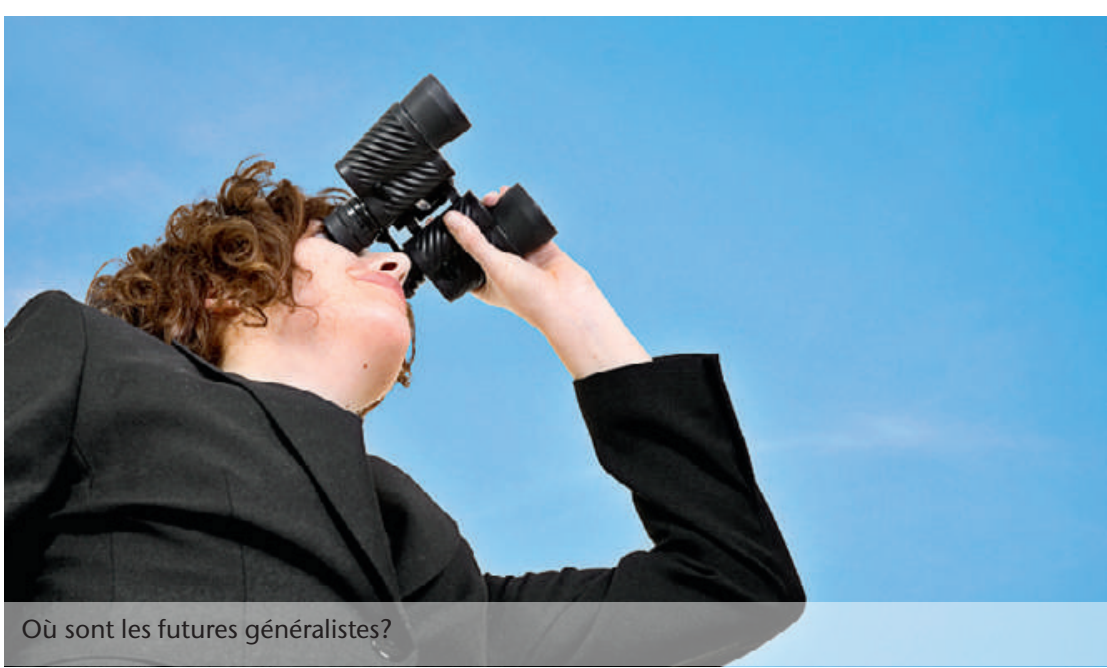

généralistes est restée relativement stable ces dernières années (de 2006 à 2011).

Age des médecins: La moyenne d'âge des médecins du secteur ambulatoire est de 53,3 ans. Depuis 2000, près de 800 médecins prennent leur retraite chaque année avec un pic de départs prévus entre 20152020. En Suisse romande, $60 \%$ des généralistes ont aujourd'hui plus de 55 ans. Considérant qu'il faut entre 10 et 15 ans pour former un médecin, il est à craindre que d'ici 2030, 40\% des consultations de médecine de premier recours ne pourront plus être assurées, selon l'Observatoire suisse de la santé (Obsan). Dans diverses spécialités, les besoins, c'està-dire la demande, dépasseront de 30\% l'offre existante (Obsan).

Répartition géographique: On constate d'importantes disparités régionales de la couverture médicale en Suisse: ainsi, Bâle et Genève connaissent la densité médicale la plus élevée du pays, alors que des régions périphériques connaissent une faible densité.

\section{Causes de la pénurie globale des médecins}

Concernant le manque global de la relève médicale (généralistes et spécialistes), on est face à une politique à court terme avec notamment un numerus clausus, explicite ou implicite, longtemps trop restrictif et à l'absence d'anticipation concernant les besoins futurs en médecins bien formés.

L'assujettissement des médecins assistants à la Loi fédérale sur le travail dès le $1^{\text {er }}$ janvier 2005 a certes eu un impact positif sur les conditions de travail des médecins en formation [5]. Or, afin de se conformer à la loi et de pallier le manque aigu de médecins hospitaliers, les hôpitaux ont rapidement dû recruter des médecins diplômés, avant tout dans les pays voisins. Ainsi, la planification du nombre de médecins à former dans nos universités a été rendue encore plus difficile et la réflexion sur l'avenir des médecins étrangers engagés dans les hôpitaux ne semble pas avoir été approfondie.

La féminisation de la profession et l'augmentation du travail partiel, $30 \%$ des femmes médecins travaillent aujourd'hui à temps partiel, contribuent également à la pénurie.

\section{Causes de l'offre insuffisante en généralistes}

Citons en premier lieu la clause du besoin: en juillet 2002, le Conseil Fédéral a édicté une ordonnance se basant sur l'article 55a de la LAMal qui stipule que l'autorisation des fournisseurs de prestations à pratiquer à la charge des assureurs peut être limitée au maximum pendant trois ans, mesure renouvelable une fois. L'introduction de cette clause a été motivée par l'objectif de prévenir une installation «massive» de médecins étrangers dans le sillage des accords bilatéraux sur la libre circulation des personnes, par crainte d'une hausse des coûts de la santé. En 2005, il $\mathrm{y}$ a eu une première prolongation du moratoire d'installation et fin 2007, le Conseil des Etats a approuvé 
une modification de la loi permettant une deuxième prolongation de la clause du besoin [6]. En 2009, une solution transitoire a été adoptée, consistant en la prolongation de la clause exclusivement pour les spécialistes [7]. Finalement, le moratoire a été abrogé le $1^{\mathrm{er}}$ janvier 2012.

Les conséquences de la clause du besoin et de son renouvellement réitéré: Pendant dix ans, toute une génération de jeunes médecins n'ont pas eu d'autres choix que de chercher une voie de spécialisation intrahospitalière. L'instauration de la clause du besoin, qui avait comme but initial de freiner l'installation de médecins étrangers, a finalement pénalisé les médecins formés en Suisse et a contribué, au moins pendant les premières années de cette mesure, au maintien du déséquilibre du ratio généralistes-spécialistes, dont nos responsables politiques s'étonnent aujourd'hui. cins par an dès 2018/2019 (contre 864 en moyenne actuellement).

Un plan stratégique de la formation médicale devrait être établi régulièrement au niveau suisse, préparant l'avenir 8-10 ans avant l'échéance, impliquant les acteurs politiques et les représentants médicaux (FMH, sociétés cantonales de médecine).

Les prérogatives des cantons pour la régulation du nombre de médecins sur leur territoire doivent être renforcées. Une planification étatique en bonne coopération avec les sociétés médicales semble une voie réaliste, afin d'équilibrer l'offre et la demande.

Afin de promouvoir la médecine générale, une amélioration et revalorisation de la formation et rétribution de cette discipline est indispensable. Plusieurs universités ont mis sur pied des instituts de médecine générale et offrent une formation complète de médecine générale avec un «label» universitaire, garant de

\section{Afin de lutter contre la pénurie de médecins, il faut avant tout investir dans la formation.}

Comble du paradoxe, en termes d'économie de santé, il ne semble pas exister de véritable étude, à notre connaissance, qui aurait examiné l'impact économique de la clause du besoin.

En 2012, après l'abrogation de la clause, les autorités cantonales ont enregistré un nombre record de demandes d'autorisation d'installation: un total de 2049 autorisations a été délivré en Suisse [8], dont 270 à Genève, 173 au Tessin et 170 dans le canton de Vaud. La grande majorité des demandes émanaient de spécialistes, dont surtout des psychiatres et des psychothérapeutes (328 demandes; 2011: 114), suivis des ophtalmologues $(131 ; 2011: 35)$ et des gynécologues (120; 2011: 49). Seulement 266 du total (2011: 224) concernaient la médecine interne générale. La majorité des spécialistes demandaient une installation dans les grandes agglomérations.

Ces chiffres montrent l'ampleur de la distorsion créée par le mauvais réglage du marché de la santé.

Une autre cause de la crise de la médecine générale est le «désamour» de la médecine de premier recours en raison d'un manque de reconnaissance et de prestige, d'une rétribution inférieure à celle des spécialistes et d'une charge de travail importante avec de nombreuses gardes.

Comment remédier à la pénurie de médecins, comment promouvoir la médecine de famille? Afin de lutter contre la pénurie de médecins, il faut avant tout investir dans la formation: la Confédération et les cantons viennent de lancer une mesure qui vise à augmenter progressivement le nombre de diplômés dès 2013, dans le but de former 1100 méde- qualité [9]. Les conditions de travail des généralistes peuvent être améliorées par le développement de nouvelles structures, tels des cabinets de groupe ou des centres de soins, qui permettent une meilleure régulation des heures de travail (et possibilité de temps partiels) et des gardes.

Finalement, dans le but d'endiguer les disparités géographiques, deux mesures, parmi d'autres, devraient être examinées: d'une part, prévoir l'obligation d'effectuer au minimum un an dans une zone périphérique lors de la formation post-graduée et d'autre part, envisager des incitations financières (aide financière à l'installation, augmentation du point TARMED) pour les médecins qui choisissent la périphérie.

En conclusion, le système de santé suisse est une construction hybride de «libre concurrence réglementée», avec de très nombreux acteurs du domaine de la politique, des assurances et des professionnels de la santé. Dans le passé, il n'y a pas eu de vision commune à moyen et long terme, mais une réactivité à court terme. Devant l'absence d'anticipation et de planification à long terme, nous sommes aujourd'hui confrontés à une démographie médicale déséquilibrée, tant en termes de pénurie de généralistes et de certains spécialistes, qu'en termes de répartition géographique.

Il faut un plan stratégique de la formation et de la couverture médicale dans notre pays, qui prépare l'avenir 8-10 ans avant l'échéance en impliquant tous les acteurs qui sont appelés à assumer leurs responsabilités, afin d'offrir à la population une médecine de haute qualité, efficace, efficiente et accessible à tous. 